\title{
Método Espectro Nodal de Grades Compostas com Aproximação Exponencial na Formulação de Ordenadas Discretas em Meios Homogêneos Não-multiplicativos
}

\section{Composite Spatial Grid Nodal Method with Exponential Approximations in Discrete Ordinate Formulation for Non-multiplying Homogeneous Mediums}

Anderson S. O. Aguiar ${ }^{1}$, Dany S. Dominguez², Susana M. Iglesias ${ }^{3}$, Carlos R. G. Hernandez 4 , Iram B. R. Ortiz ${ }^{5}$.

\section{RESUMO}

Problemas de transporte de nêutrons em meios não multiplicativos aparecem em diversas aplicações de ciências e engenharias, com destaque para o desenho de estruturas de blindagem. Neste trabalho apresentamos o método espectro nodal de grades compostas com aproximação exponencial para problemas de transporte de nêutrons em meios homogêneos não multiplicativos. Usamos como modelo matemático a formulação de ordenadas discretas em geometria bidimensional cartesiana em aproximação de uma velocidade com fonte de espalhamento isotrópica. Como em outros métodos de grades compostas a discretização é feita em duas etapas, e uma função exponencial é usada para aproximar os termos de fuga transversal na região. O mecanismo de discretização transforma o problema bidimensional em dois problemas unidimensionais acoplados que são resolvidos a partir de um esquema de direções alternadas. Resultados numéricos são oferecidos para um problema modelo, mostrando que o método proposto gera resultados precisos com baixo custo computacional.

Palavras-chave: Aproximação exponencial. Grades compostas. Ordenadas discretas. Meios não-multiplicativos.

\section{ABSTRACT}

Neutron transport problems in non-multiplying media appear in several applications in science and engineering, with emphasis on the design of shielding structures. In this work, we present the composite spatial grids spectral nodal method with an exponential approximation for neutron transport problems in homogeneous nonmultiplying media. We use as a mathematical model the discrete ordinates formulation in two-dimensional Cartesian geometry with one-speed approximation and isotropic scattering source. The discretization process is done in two steps like is as composite spatial grid methods. An exponential function is used to approximate the transverse leakage terms in the region. The multi-step discretization mechanism transforms the two-dimensional problem into two coupled one-dimensional problems, that are solved using the alternative directions iterative scheme. Numerical results are offered for a model problem, showing that the proposed method generates accurate results with a low computational cost.

Keywords: Exponential approximation. Composite spatial grids. Discrete ordinates. Non-multiplying mediums

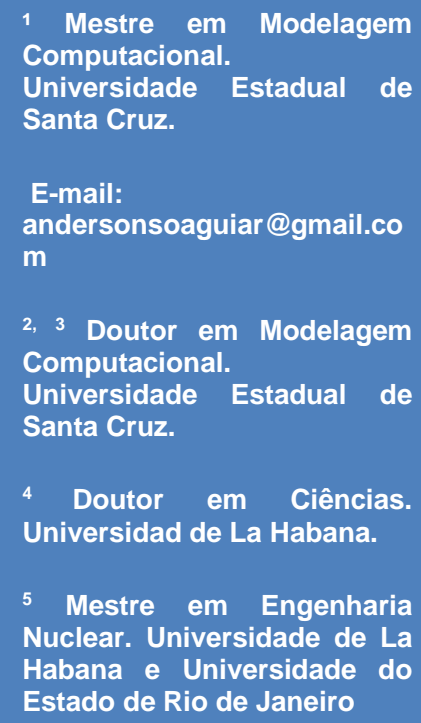




\section{INTRODUCÃO}

A modelagem computacional da migração de nêutrons em um meio material desperta interesse em diversas áreas de engenharia, com aplicações nos cálculos de blindagem de radiação (DOMINGUEZ; BARROS, 2007), na exploração de reatores nucleares (DOMINGUEZ, et al., 2018), nas terapias baseadas em nêutrons (MOSS, 2014), e na indústria de petróleo e gás com o uso de sondas neutrônicas (FRANKLE; DALE, 2013). Uma das alternativas mais utilizadas é na modelagem matemática deste problema é a formulação de ordenadas discretas $\left(S_{N}\right)$ da equação de transporte de nêutrons (LEWIS; W. F. MILLER, 1984). O desenvolvimento de métodos numéricos para resolver este problema tem motivado diversos pesquisadores nas últimas décadas. Por razões de eficiência computacional um dos principais objetivos é obter métodos que gerem soluções numéricas precisas utilizando malhas espaciais grossas.

Em geometria $X, Y$ a família de métodos espectro-nodais tem obtido bons resultados, tendo como principais características o tratamento analítico dos termos de fonte de espalhamento, aproximando apenas os termos de fuga transversal. O primeiro desta família foi o método espectro-nodal com aproximação constante proposto por (BARROS; LARSEN, 1992). Propostas mais recentes buscaram aprimorar a aproximação utilizada, com destaque para o método espectro-nodal com aproximação exponencial (DE MELLO; BARROS, 2002) e o método espectro-nodal com aproximação linear (DOMINGUEZ, Dany S.; BARROS, 2007). Estes métodos tinham como principais desvantagens a complexidade algébrica associada a obtenção das equações de varredura, e a dificuldade de implementação do algoritmo computacional, ambas causadas pelo uso da varredura de inversão nodal. Por outro lado, estes métodos apresentam baixa sensibilidade as dimensões da grade espacial e geram resultados numéricos precisos em malhas grossas.

Os métodos de espectro-nodais de grades compostas (CSG-SGF, Composite Spatial Grids - Spectral Green's Function) surgem como uma alternativa aos métodos espectro-nodais, visando aproveitar a capacidade destes de gerar soluções precisas em malhas grossas, ao mesmo tempo em que amenizam sua complexidade algébrica e computacional. Os métodos de grades compostas foram propostos incialmente por (DOMINGUEZ, Dany S et al., 2010) através do método CSG-SGF com aproximação constante para problemas de fonte fixa na formulação $S_{N}$ (CSG-SGF-CN, Composite Spatial Grids - Spectral Green's Function - Constant Nodal). Houveram avanços nesta 
família de métodos com o método de grades compostas para problemas de autovalor na aproximação de difusão (NASCIMENTO; DOMINGUEZ, 2015), e o método de grades compostas para problemas de autovalor na formulação $S_{N}$ (DOMINGUEZ, Dany S. et al., 2018), ambos os métodos também utilizaram aproximação constante nos termos de fuga em nível de região.

Neste trabalho apresentamos o método espectro-nodal de grades compostas com aproximação exponencial para problemas $S_{N}$ homogêneos de fonte fixa em geometria $X, Y$ com fonte de espalhamento isotrópica e um grupo de energia (CSG-SGF-Exp, Composite Spatial Grid - Spectral Green's Function - Exponential). O trabalho representa um novo avanço nos métodos de grades compostas, sendo o primeiro desta família em utilizar a aproximação exponencial. A discretização do domínio de cálculo é feita em duas etapas, na primeira utilizamos uma malha grossa em nível de região, na segunda usamos duas grades finas independentes, uma para cada direção espacial. O procedimento de discretização transforma o problema bidimensional descrito por um sistema de equações em derivadas parciais em dois problemas de equações diferenciais ordinárias, um para cada direção espacial, que podem ser resolvidos de forma independente usando uma esquema iterativo explicito de direções alternadas (BIRKHOFF; VARGA; YOUNG, 1962). Para ilustrar a precisão e o desempenho do método proposto são oferecidos resultados numéricos para um problema modelo.

Na próxima seção oferecemos os fundamentos matemáticos do método CSG-SGFExp. Na seção 3, apresentamos os resultados numéricos e a influência neles das dimensões da grade espacial. Por últimos na seção 4 , oferecemos as conclusões do trabalho e sugestões de trabalhos futuros.

\section{FUNDAMENTOS DO METODO EXPONENCIAL DE GRADES COMPOSTAS}

O método-espectro nodal de grades compostas com aproximação exponencial (CSG-SGF-Exp) combina a discretização em duas etapas dos métodos de grades compostas (DOMINGUEZ, Dany $S$ et al., 2010) e o uso da aproximação exponencial proposta por (DE MELLO; BARROS, 2002), em particular a aproximação exponencial é utilizada para aproximar os termos de fuga transversal na região. Para obtermos as equações discretizadas de varredura do método CSG-SGF-Exp partimos da formulação de ordenadas discretas em aproximação de uma velocidade em geometria $X, Y$ e com 
fonte de espalhamento isotrópica. Considerando um domínio retangular de comprimento $X$ e largura $Y$, a formulação $\mathrm{S}_{N}\left(\mathrm{CARLSON}\right.$; LATHROP, 1968) para uma região $R_{i j}$ com propriedades materiais constantes aparece na forma

$$
\begin{gathered}
\mu_{m} \frac{\partial}{\partial x} \psi_{m}(x, y)+\eta_{m} \frac{\partial}{\partial y} \psi_{m}(x, y)+\sigma_{t_{i j}}(x, y) \psi_{m}(x, y)= \\
\frac{\sigma_{s 0_{i j}}(x, y)}{4} \sum_{n=1}^{M} \psi_{n}(x, y) \omega_{n}+Q_{m i j}(x, y)
\end{gathered}
$$

onde $(x, y) \in R_{i j}$, os cosenos diretores $\mu_{m}$ e $\eta_{m}$ representam a direção angular $m$, $m=1, \ldots, M ; \psi_{m}(x, y)$ o fluxo angular de nêutrons; $\sigma_{t_{i j}}$ a seção de choque total; $\sigma_{s 0_{i j}}$ a seção diferencial de espalhamento; $Q_{m i j}$ a fonte externa de neutrôns e $\omega_{n}$ os pesos da quadratura angular de simetria de níveis (LEWIS; W. F. MILLER, 1984). Neste trabalho consideramos apenas a condição de contorno de tipo vácuo que pode ser expressa para as direções incidentes na fronteira do domínio como

$$
\begin{array}{ll}
\psi_{m}(0, y)=0, & \mu_{m}>0 \\
\psi_{m}(X, y)=0, & \mu_{m}<0, \\
\psi_{m}(x, 0)=0, & \eta_{m}>0 \\
\psi_{m}(x, Y)=0, & \eta_{m}<0 .
\end{array}
$$

No método de grades compostas, o primeiro passo envolve discretizar o domínio utilizando uma malha grossa $\Lambda$, de forma que cada região $R_{i j},(x, y) \in R_{i j}, x_{i-1 / 2} \leq x \leq x_{i+1 / 2}$, $y_{y-1 / 2} \leq y \leq y_{j+1 / 2}$, coincida com uma célula da discretização, conforme pode ser observado na Figura 1a. Em seguida sobre a grade $\Lambda$ são obtidas as equações $S_{N}$ integradas transversalmente para cada uma das direções espaciais $x$ e $y$. A seguir, ilustramos os fundamentos matemáticos do método CSG-SGF-Exp para a direção espacial $x$, o mesmo procedimento deve ser acompanhado para a direção espacial $y$.

Obtemos a equação $S_{N}$ integrada transversalmente dependente de $x$, integrando a eq. (1) em $R_{i j}$ na direção $y$, o que corresponde a aplicar o operador

$$
\frac{1}{L_{y j}} \int_{y_{j-1 / 2}}^{y_{j+1 / 2}} \sqsubset d y,
$$

o resultado aparece na forma 
AGUIAR, A.S. O; DOMINGUEZ, D.S; IGLESIAS, S.M; HERNANDEZ, C.R.G.

Método Espectro Nodal de Grades Compostas com Aproximação Exponencial na Formulação de Ordenadas Discretas em Meios Homogêneos Não-multiplicativos.

$$
\begin{aligned}
& \mu_{m} \frac{d}{d x} \tilde{\psi}_{m j}(x)+\sigma_{t_{i j}} \tilde{\psi}_{m j}(x)= \\
& \frac{\sigma_{s 0_{i j}}}{4} \sum_{n=1}^{M} \tilde{\psi}_{n j}(x) \omega_{n}+Q_{m i j}-\frac{\eta_{m}}{L_{y j}}\left[\psi_{m}\left(x, y_{j+1 / 2}\right)-\psi_{m}\left(x, y_{j-1 / 2}\right)\right]^{\prime}
\end{aligned}
$$

onde definimos o fluxo angular médio integrado em $y, \tilde{\psi}_{m j}(x)$, como

$$
\tilde{\psi}_{m j}(x)=\frac{1}{L_{y j}} \int_{y_{j-1 / 2}}^{y_{j+1 / 2}} \psi_{m}(x, y) d y .
$$

(c)

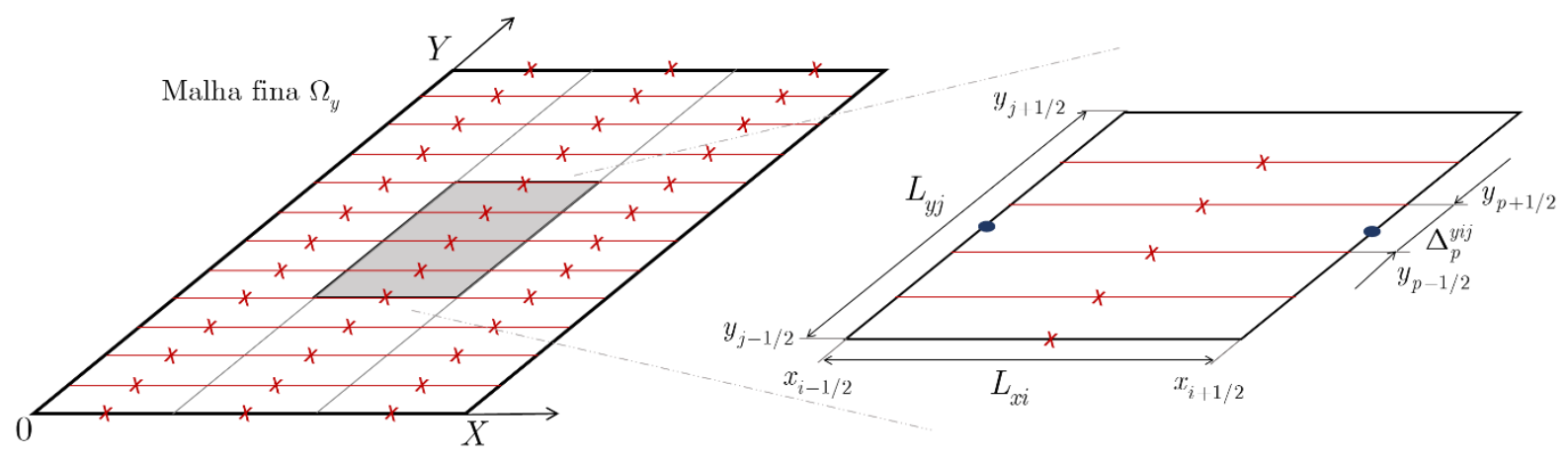

(b)



(a)

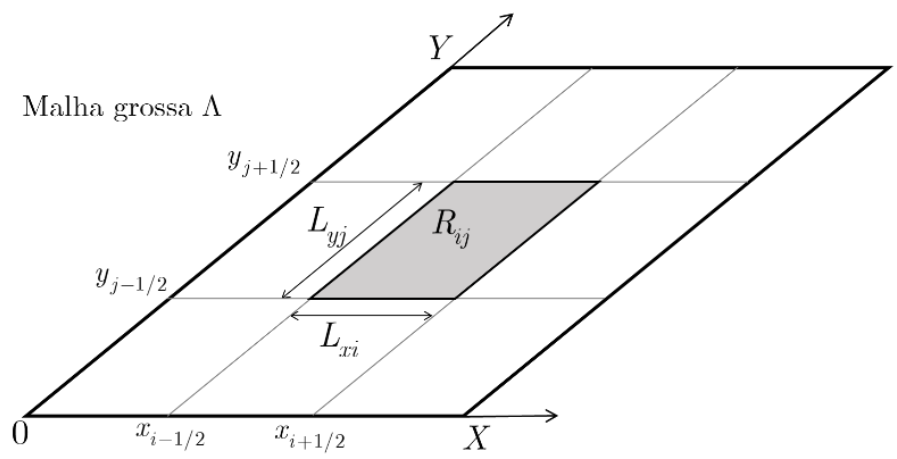

Figura 1. Malha espacial composta (a) Malha grossa por região, (b) Malha fina na direção $x$, e (c) Malha fina na direção $y$. 
O processo de integração transversal em nível de região conduz a um sistema de $2 M$ equações ( $M$ equações da direção $x$ eq. (4), e $M$ equações da direção $y$ ), e $4 M$ incógnitas que envolvem os fluxos médios na região, e os fluxos nas arestas $\left[\tilde{\psi}_{m j}(\mathrm{x})\right.$, $\hat{\psi}_{m i}(y), \psi_{m}\left(x_{i \pm 1 / 2}, y\right)$ e $\left.\psi_{m}\left(x, y_{j \pm 1 / 2}\right)\right]$. Por isso, para obtermos solução única é preciso introduzir aproximações para os termos de fuga transversal ao longo das arestas da região.

Neste trabalho consideramos que os fluxos nas arestas apresentam um decaimento exponencial, conforme (DE MELLO; BARROS, 2002) estas aproximações aparecem como

$$
\psi_{m}\left(x, y_{i \pm 1 / 2}\right) \cong N_{i j}^{x} \hat{\psi}_{m i j \pm 1 / 2} e^{-\lambda_{i j}\left(x-x_{i}\right)},
$$

sendo $x_{i}=\left(x_{i+1 / 2}-x_{i-1 / 2}\right) / 2$ e $\hat{\psi}_{m i j \pm 1 / 2}=\hat{\psi}_{m i}\left(y_{j \pm 1 / 2}\right)$. O parâmetro $\lambda_{i j}$ é aproximado pela seção de choque de absorção na região conforme

$$
\lambda \equiv\left\{\begin{array}{l}
\sigma_{a}, \mu_{m}>0 \\
-\sigma_{a}, \mu_{m}<0
\end{array}\right\} .
$$

Por outro lado, o parâmetro $N_{i j}^{x}$ é calculado de modo que a aproximação (6) satisfaça o operador integral (3), o que nos leva a

$$
N_{i j}^{x}=\frac{L_{x i} \lambda_{i j}}{2}\left[\operatorname{senh}\left(\frac{L_{x i} \lambda_{i j}}{2}\right)\right]^{-1} .
$$

Introduzindo a aproximação (6) na integrada transversalmente eq. (4), obtemos

$$
\mu_{m} \frac{d}{d x} \tilde{\psi}_{m j}(x)+\sigma_{t i j} \tilde{\psi}_{m j}(x)=\frac{\sigma_{s 0 i j}}{4} \sum_{n=1}^{M} \tilde{\psi}_{n j}(x) \omega_{n}+Q_{m i j}-N_{i j}^{x} \hat{\tau}_{m i j} e^{-\lambda_{i j}\left(x-x_{i}\right)},
$$

onde $\hat{\tau}_{m i j}$ representa a fuga transversal na região, sendo definida na forma

$$
\hat{\tau}_{m i j}=\frac{\eta_{m}}{L_{y j}}\left[\hat{\psi}_{m i j+1 / 2}-\hat{\psi}_{m i j-1 / 2}\right] .
$$

A equação integrada transversalmente com aproximação exponencial nos termos de fuga eq. (9), e sua correspondente dependente da direção y representam dois problemas unidimensionais de equações diferenciais ordinárias (EDOs). Desta forma, a través do procedimento de integração transversal temos transformado o problema bidimensional em derivadas parciais eq. (1), em dois problemas unidimensionais de EDOs. Os problemas 
unidimensionais mesmo que acoplados pelos termos de fuga transversal podem ser resolvidos separadamente usando um esquema iterativo de direções alternadas.

Em seguida obtemos a solução analítica geral do problema unidimensional dependente de $x$, a mesma apresenta uma componente homogênea que calculamos utilizando a técnica de análises espectral (CASE; ZWEIFEL, 1967), e uma componente particular que é calculada usando o método dos coeficientes a determinar (ARNOLD, 1992). A solução analítica geral da eq. (9) aparece como

$$
\tilde{\psi}_{m j}(x)=\sum_{l=1}^{M} \alpha_{l}^{x} a_{m}^{x}\left(v_{l}\right) e^{\left(\sigma_{t i j} x / v_{l}\right)}+\frac{Q_{i j}}{\sigma_{t_{i j}}-\sigma_{s 0_{i j}}}-e^{-\lambda_{i j}\left(x-x_{i}\right)} \hat{\Theta}_{m i j},
$$

onde $\alpha_{l}^{x}$ são constantes arbitrárias, $a_{m}^{x}$ e $v_{l}$ são os respectivos autovetores e autovalores locais associados ao problema homogêneo e o parâmetro $\hat{\Theta}_{m i j}$ é definido pela expressão

$$
\hat{\Theta}_{m i j}=\frac{N_{i j}^{x}}{\sigma_{t_{i j}}-\lambda_{i j} \mu_{m}}\left[\hat{\tau}_{m i j}-\frac{\frac{\sigma_{s 0_{i j}}}{4} \sum_{n=1}^{M} \frac{\hat{\tau}_{n i j} \omega_{n}}{\sigma_{t_{i j}}-\lambda_{i j} \mu_{m}}}{1-\frac{\sigma_{s 0_{i j}}}{4} \sum_{n=1}^{M} \frac{\omega_{n}}{\sigma_{t_{i j}}-\lambda_{i j} \mu_{m}}}\right] .
$$

Uma descrição detalhada do procedimento para obter a solução analítica geral das equações integradas transversalmente pode ser encontrada em (OLIVEIRA AGUIAR, 2016).

Neste ponto obtemos as equações de balanço espacial para os problemas unidimensionais, eq. (9). Para isso, introduzimos a segunda etapa de discretização, onde as malhas finas $\Omega_{x}$ e $\Omega_{y}$ devem ser consideradas para cada direção espacial no interior da região $R_{i j}$ (vide Figura 1a e b). Para obter a equação $\mathrm{S}_{N}$ discretizada de balanço espacial na direção $x$, dividimos as regiões $R_{i j}$ em $K$ células de comprimento $\Delta_{k}^{x i j}$, conforme a Figura 1b, e aplicamos o operador

$$
\frac{1}{\Delta_{k}^{x i j}} \int_{x_{k-1 / 2}}^{x_{k+1 / 2}} \cdot d x
$$

na eq. (9), o resultado aparece como

$$
\frac{\mu_{m}}{\Delta_{k}^{x i j}}\left(\tilde{\psi}_{m k+1 / 2 j}-\tilde{\psi}_{m k-1 / 2 j}\right)+\sigma_{t_{i j}} \bar{\psi}_{m k j}^{x}=\frac{\sigma_{s 0_{i j}}}{4} \sum_{n=1}^{M} \bar{\psi}_{n k j}^{x} \omega_{n}+Q_{i j}-\frac{2 N_{i j}^{x} \operatorname{senh}\left(\frac{\lambda_{i j} \Delta_{k}^{x i j}}{2}\right) \hat{\tau}_{m i j}}{\lambda_{i j} \Delta_{k}^{x i j}},
$$


onde definimos o fluxo médio no nodo retangular $\Omega_{x k}, x_{k-1 / 2} \leq x \leq x_{k+1 / 2}, \quad y_{j-1 / 2} \leq y \leq y_{j+1 / 2}$, $\bar{\psi}_{m k j}^{x}$, como

$$
\bar{\psi}_{m k j}^{x}=\frac{1}{\Delta_{k}^{x i j} L_{y j}} \int_{x_{k-1 / 2}}^{x_{k+1 / 2}} \int_{y_{j-1 / 2}}^{y_{j+1 / 2}} \psi_{m}(x, y) d y d x .
$$

Para cada célula da malha fina $\Omega_{x}$ a eq. (14) representa um sistema de $M$ equações lineares e algébricas com $2 M$ incógnitas $\left(\tilde{\psi}_{m k \pm 1 / 2 j}\right.$ e $\left.\bar{\psi}_{m k j}^{x}\right)$, sendo um sistema indeterminado. A fim de obter solução única para o problema precisamos incluir $M$ equações auxiliares, além de considerar as condições de contorno nas fronteiras do domínio e de continuidade entre as interfaces das células. A equação auxiliar do método CSG-SGF-Exp é semelhante à equação auxiliar do método SGF-CN (BARROS; LARSEN, 1992) e aparece como

$$
\bar{\psi}_{m k j}^{x}=\sum_{\mu_{n}>0} \theta_{m n} \tilde{\psi}_{n k-1 / 2 j}+\sum_{\mu_{n}<0} \theta_{m n} \tilde{\psi}_{n k+1 / 2 j}+H_{m k j}^{y},
$$

Onde os parâmetros $\theta_{m n}$ e $H_{m i j}^{y}$ devem ser calculados de forma a preservarem as componentes homogênea e particular da solução analítica geral eq. (11), respetivamente. Os detalhes relativos ao cálculo do espectro e dos parâmetros $\theta_{m n}$ da equação auxiliar podem ser encontrados em (DOMINGUEZ, Dany S et al., 2010). O parâmetro $H_{m i j}^{y}$ aparece na forma

$$
\begin{aligned}
& H_{m k j}^{y}=\left(1-\sum_{n=1}^{M} \theta_{m n}\right) \frac{Q_{i j}}{\sigma_{t_{i j}}-\sigma_{s 0_{i j}}}+e^{-\lambda_{i j}\left(x_{k-1 / 2}-x_{i}\right)}\left(\sum_{\mu_{n}>0} \theta_{m n} \hat{\Theta}_{n i j}+\frac{\hat{\Theta}_{m i j}}{\Delta_{k}^{x i j} \lambda_{i j}}\right) . \\
& +e^{-\lambda_{i j}\left(x_{k+1 / 2}-x_{i}\right)}\left(\sum_{\mu_{n}<0} \theta_{m n} \hat{\Theta}_{n i j}-\frac{\hat{\Theta}_{m i j}}{\Delta_{k}^{x i j} \lambda_{i j}}\right),
\end{aligned}
$$

Neste ponto para o problema associado à direção $x$, temos as equações discretizadas de balanço espacial (14), as equações auxiliares (16) e as condições de contorno (2), que formam um sistema solúvel e determinado para o problema unidimensional. Para implementarmos um esquema iterativo que gere uma solução numérica, devemos obter a equação de varredura correspondente, onde o fluxo emergente em cada célula é calculado em função dos fluxos incidentes. Obtemos a equação de varredura na direção $x$ substituindo a eq. (16) na eq. (14) e isolamos os fluxos emergentes em cada direção, o que nos leva a 


$$
\tilde{\psi}_{m k \pm 1 / 2 j}=\tilde{\psi}_{m k+1 / 2 j}+\frac{\Delta_{k}^{x i j} \sigma_{t_{i j}}}{\left|\mu_{m}\right|}\left\{\begin{array}{l}
\frac{Q_{i j}}{\sigma_{t_{i j}}}-\frac{2 N_{i j}^{x} \operatorname{senh}\left(\lambda_{i j} \Delta_{k}^{x i j} / 2\right) \hat{\tau}_{m i j}}{\lambda_{i j} \Delta_{k}^{x i j} \sigma_{t_{i j}}}-H_{m k j}^{y} \\
+\frac{c_{0_{i j}}}{4} \sum_{n=1}^{M} H_{n k j}^{y} \omega_{n}+\sum_{\mu_{n}>0}\left(\frac{c_{0_{i j}}}{4} \mathrm{~T}_{n}^{\theta}-\theta_{m n}\right) \tilde{\psi}_{n k-1 / 2 j} \\
+\sum_{\mu_{n}<0}\left(\frac{c_{0_{i j}}}{4} \mathrm{~T}_{n}^{\theta}-\theta_{m n}\right) \tilde{\psi}_{n k+1 / 2 j}
\end{array}\right\}, \begin{aligned}
& \mu_{m}>0 \\
& \mu_{m}<0
\end{aligned},
$$

Onde definimos $\mathrm{T}_{n}^{\theta}=\sum_{m=1}^{M} \theta_{m n} \omega_{m}$. A expressão (18) representa duas equações, uma para calcular os fluxos emergentes na aresta direita da célula $\tilde{\psi}_{m k+1 / 2 j}$ isto é as direções angulares com $\mu_{m}>0$; e a outra equação para calcular os fluxos emergentes na aresta esquerda da célula $\tilde{\psi}_{m k-1 / 2 j}$, ou seja, as direções angulares com $\mu_{m}<0$.

Analisando a equação de varredura (18) constatamos a presença do coeficiente $H_{m i j}^{y}$ definido pela eq. (17), onde temos a presença do termo exponencial $e^{-\lambda_{i j}\left(x_{k \pm 1 / 2}-x_{i}\right)}$ que introduz instabilidade numérica nos cálculos devido a mudança de sinal da exponencial ao percorrer as células da grade espacial interna em uma região. Para mitigar essa instabilidade foram consideradas quatro estratégias numéricas para avaliar os termos exponenciais, as mesmas são resumidas na Tabela 1.

Tabela 1. Aproximações do termo exponencial para o problema unidimensional na direção $x$.

\begin{tabular}{clc}
\hline Aproximação & \multicolumn{1}{c}{ Descrição } & Definição \\
\hline 1 & $\begin{array}{l}\text { Cálculo exato das distâncias, } \\
\text { definição matemática }\end{array}$ & $e^{-\lambda_{i j}\left(x_{k \pm 1 / 2}-x_{i}\right)}=e^{-\lambda_{i j}\left(x_{k \pm 1 / 2}-x_{i}\right)}$ \\
\hline 2 & $\begin{array}{l}\text { Aproxima pela metade da largura } \\
\text { da região }\end{array}$ & $e^{-\lambda_{i j}\left(x_{k \pm 1 / 2}-x_{i}\right)}=e^{-\lambda_{i j} L_{x i} / 2}$ \\
\hline 3 & $\begin{array}{l}\text { Aproxima por um quarto da } \\
\text { largura da região }\end{array}$ & $e^{-\lambda_{i j}\left(x_{k \pm 1 / 2}-x_{i}\right)}=e^{-\lambda_{i j} L_{x i} / 4}$ \\
\hline 4 & $\begin{array}{l}\text { Aproxima pela largura da célula } \\
\text { da malha fina }\end{array}$ & $e^{-\lambda_{i j}\left(x_{k \pm 1 / 2}-x_{i}\right)}=e^{-\lambda_{i j} \Delta_{k}^{x i j}}$ \\
\hline
\end{tabular}

O processo de obter as equações de varredura do método CSG-SGF-Exp envolve duas etapas de discretização. Na primeira usamos uma grade grossa em nível de região material, aplicamos o conceito de integração transversal reduzindo o problema original a dois problemas unidimensionais de EDOs. Na segunda etapa utilizamos uma malha fina no interior de cada região para obtermos as equações de balanço espacial, e 
introduzimos as equações auxiliares. A combinação das equações de balanço com as auxiliares nos conduz a uma equação de varredura que pode ser utilizada para calcular os fluxos emergentes em cada direção angular. A equação de varredura na direção $x$, eq. (18), e sua correspondente na direção y são utilizadas em um esquema de direções alternadas explícito, onde as fugas são atualizadas em cada iteração, convergindo à solução do problema de ordenadas discretas. $O$ algoritmo de direções alternadas é similar ao utilizado em outros métodos de grades múltiplas e pode ser verificado em (DOMINGUEZ, D.S.; HERNANDEZ; BARROS, 2010; DOMINGUEZ, Dany S. et al., 2018).

\section{RESULTADOS NUMERICOS E DISCUSSÄO}

Para validar a formulação numérica proposta neste trabalho para o método espectronodal de grades compostas com aproximação exponencial resolvemos um problema modelo homogêneo reportado em (DOMINGUEZ, Dany S et al., 2010). As dimensões do problema, suas condições de contorno e parâmetros materiais são oferecidos na Figura 2, a ordem de quadratura considerada nos experimentos numéricos foi de $\mathrm{N}=4$, e o critério para encerrar o processo iterativo foi que o máximo desvio relativo entre duas iterações sucessivas do fluxo angular não ultrapasse 1E-6.

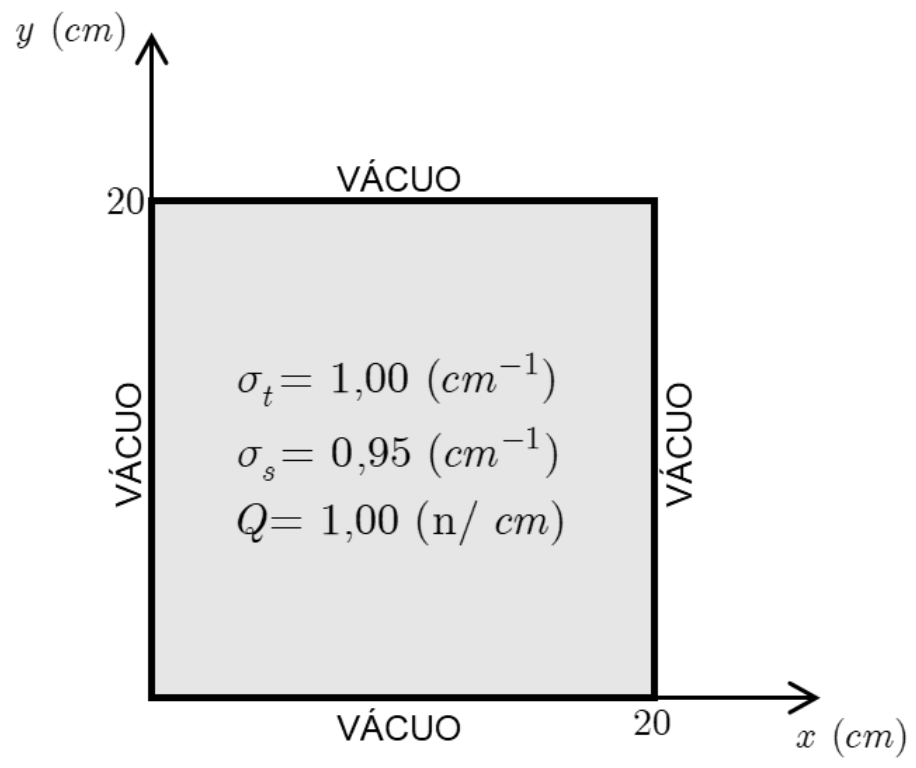

Figura 2. Problema modelo

Nos problemas de blindagem uma das grandezas que gera maior interesse é a fuga de nêutrons pelas fronteiras do domínio, considerando a simetria do problema em relação 
aos eixos centrais do domínio as fugas pelas quatro fronteiras são iguais, as fugas pela fronteira superior $\left(J_{S}\right)$ e direita $\left(J_{D}\right)$ podem ser estimadas pelas expressões

$$
\begin{aligned}
& J_{S}=\frac{1}{2} \underset{h_{n}>0}{\stackrel{\mathrm{a}}{h_{n}}} h_{n} \hat{y}_{n, 1, J+1 / 2} L_{x} w_{n}, \\
& J_{D}=\frac{1}{2} \underset{m_{n}>0}{\stackrel{\circ}{a}} m_{n} y_{n, I+1 / 2,1}^{\%} L_{y} w_{n} .
\end{aligned}
$$

$\mathrm{Na}$ Tabela 2 oferecemos os resultados numéricos gerados pelo método CSG-SGFExp para o problema modelo da Figura 2, considerando as aproximações do termo exponencial (vide Tabela 1) e diversas configurações de malha. Incluímos para fins de comparação os valores gerados pelo método de grades compostas com aproximação constante $(\mathrm{CN})$. Adicionalmente, na Figura 3 mostramos os desvios relativos para o fluxo médio no domínio e as fugas considerando como referência os valores gerados pelos métodos LN (BADRUZZAMAN, 1990) e SGF-LN (DOMINGUEZ, Dany S.; BARROS, 2007).

Tabela 2. Resultados numéricos do problema modelo para diferentes aproximações do termo exponencial e grade grossa de uma região.

\begin{tabular}{cccccc}
\hline Malha & Aprox. Exp. & $\bar{f}_{R}$ & $J_{D}$ & $J_{S}$ & Iterações \\
\hline \multirow{4}{*}{$1 \times 4 \times 4$} & $\left(x-x_{i}\right)$ & $1,3400 \mathrm{E}+01$ & $6,6919 \mathrm{E}+01$ & $6,7646 \mathrm{E}+01$ & 17 \\
& $L_{x i} / 2$ & $1,3975 \mathrm{E}+01$ & $5,0415 \mathrm{E}+01$ & $5,1149 \mathrm{E}+01$ & 25 \\
& $L_{x i} / 4$ & $1,3966 \mathrm{E}+01$ & $5,6145 \mathrm{E}+01$ & $5,6548 \mathrm{E}+01$ & 22 \\
& $h_{x}$ & $1,3966 \mathrm{E}+01$ & $5,6145 \mathrm{E}+01$ & $5,6548 \mathrm{E}+01$ & 22 \\
& $\mathrm{CN}$ & $1,3232 \mathrm{E}+01$ & $6,7679 \mathrm{E}+01$ & $6,7679 \mathrm{E}+01$ & 16 \\
\hline \multirow{4}{*}{$1 \times 8 \times 8$} & $\left(x-x_{i}\right)$ & $1,3359 \mathrm{E}+01$ & $6,7580 \mathrm{E}+01$ & $6,7949 \mathrm{E}+01$ & 21 \\
& $L_{x i} / 2$ & $1,3651 \mathrm{E}+01$ & $5,6214 \mathrm{E}+01$ & $5,6721 \mathrm{E}+01$ & 23 \\
& $L_{x i} / 4$ & $1,3641 \mathrm{E}+01$ & $6,0700 \mathrm{E}+01$ & $6,0991 \mathrm{E}+01$ & 23 \\
& $h_{x}$ & $1,3639 \mathrm{E}+01$ & $6,2807 \mathrm{E}+01$ & $6,2998 \mathrm{E}+01$ & 23 \\
& $\mathrm{CN}$ & $1,3232 \mathrm{E}+01$ & $6,7679 \mathrm{E}+01$ & $6,7679 \mathrm{E}+01$ & 20 \\
\hline \multirow{4}{*}{$1 \times 16 \times 16$} & $\left(x-x_{i}\right)$ & $1,3345 \mathrm{E}+01$ & $6,7852 \mathrm{E}+01$ & $6,7987 \mathrm{E}+01$ & 27 \\
& $L_{x i} / 2$ & $1,3528 \mathrm{E}+01$ & $5,9781 \mathrm{E}+01$ & $6,0122 \mathrm{E}+01$ & 29 \\
& $L_{x i} / 4$ & $1,3518 \mathrm{E}+01$ & $6,3088 \mathrm{E}+01$ & $6,3298 \mathrm{E}+01$ & 28 \\
& $h_{x}$ & $1,3515 \mathrm{E}+01$ & $6,5392 \mathrm{E}+01$ & $6,5512 \mathrm{E}+01$ & 28 \\
& $\mathrm{CN}$ & $1,3232 \mathrm{E}+01$ & $6,7679 \mathrm{E}+01$ & $6,7679 \mathrm{E}+01$ & 27 \\
\hline
\end{tabular}




\begin{tabular}{|c|c|c|c|c|c|}
\hline & $L_{x i} / 2$ & $1,3508 E+01$ & $6,0547 E+01$ & $6,0854 \mathrm{E}+01$ & 31 \\
\hline \multirow[t]{5}{*}{$1 \times 20 \times 20$} & $L_{x i} / 4$ & $1,3498 E+01$ & $6,3566 \mathrm{E}+01$ & $6,3760 \mathrm{E}+01$ & 30 \\
\hline & $h_{x}$ & $1,3495 E+01$ & $6,5802 E+01$ & $6,5913 E+01$ & 30 \\
\hline & $\mathrm{CN}$ & $1,3232 \mathrm{E}+01$ & $6,7679 \mathrm{E}+01$ & $6,7679 E+01$ & 29 \\
\hline & $\left(x-x_{i}\right)$ & 1,3334E+01 & $6,8022 \mathrm{E}+01$ & $6,8048 E+01$ & 36 \\
\hline & $L_{x i} / 2$ & $1,3474 \mathrm{E}+01$ & $6,2127 \mathrm{E}+01$ & $6,2375 E+01$ & 37 \\
\hline \multirow[t]{5}{*}{$1 \times 40 \times 40$} & $L_{x i} / 4$ & $1,3464 \mathrm{E}+01$ & $6,4515 E+01$ & $6,4680 \mathrm{E}+01$ & 37 \\
\hline & $h_{x}$ & 1,3461E+01 & $6,6488 \mathrm{E}+01$ & $6,6586 E+01$ & 37 \\
\hline & $\mathrm{CN}$ & 1,3232E+01 & $6,7679 \mathrm{E}+01$ & $6,7679 \mathrm{E}+01$ & 36 \\
\hline & $\left(x-x_{i}\right)$ & 1,3329E+01 & $6,8081 E+01$ & $6,8086 E+01$ & 42 \\
\hline & $L_{x i} / 2$ & $1,3460 \mathrm{E}+01$ & $6,2940 \mathrm{E}+01$ & $6,3168 E+01$ & 43 \\
\hline \multirow[t]{3}{*}{$1 \times 80 \times 80$} & $L_{x i} / 4$ & $1,3450 \mathrm{E}+01$ & $6,4985 E+01$ & $6,5140 \mathrm{E}+01$ & 42 \\
\hline & $h_{x}$ & $1,3446 \mathrm{E}+01$ & $6,6756 \mathrm{E}+01$ & $6,6850 E+01$ & 42 \\
\hline & $\mathrm{CN}$ & $1,3232 E+01$ & $6,7679 \mathrm{E}+01$ & $6,7679 E+01$ & 42 \\
\hline- & Referência & $1,3034 \mathrm{E}+01$ & $7,1753 \mathrm{E}+01$ & $7,1753 \mathrm{E}+01$ & - \\
\hline
\end{tabular}

1×4x4 - 1 células na malha grossa , 4 células na malha fina em $x, 4$ células na malha fina em $y$ $\left(x-x_{i}\right), L_{x i} / 2, L_{x i} / 4$ e $h_{x}$ - são respetivamente as aproximações exponencias da Tabela 1

$\overline{f_{R}}$ - fluxo escalar de nêutrons na região $\mathrm{R}$

$J_{D}, J_{S}$ - corrente de nêutrons (fugas) nas fronteiras direita (D) e superior (S)

$\mathrm{CN}$ - resultados gerados pelo método CSG-SGF-CN

Referência - valor de referência obtido pelos métodos LN e SGF-LN
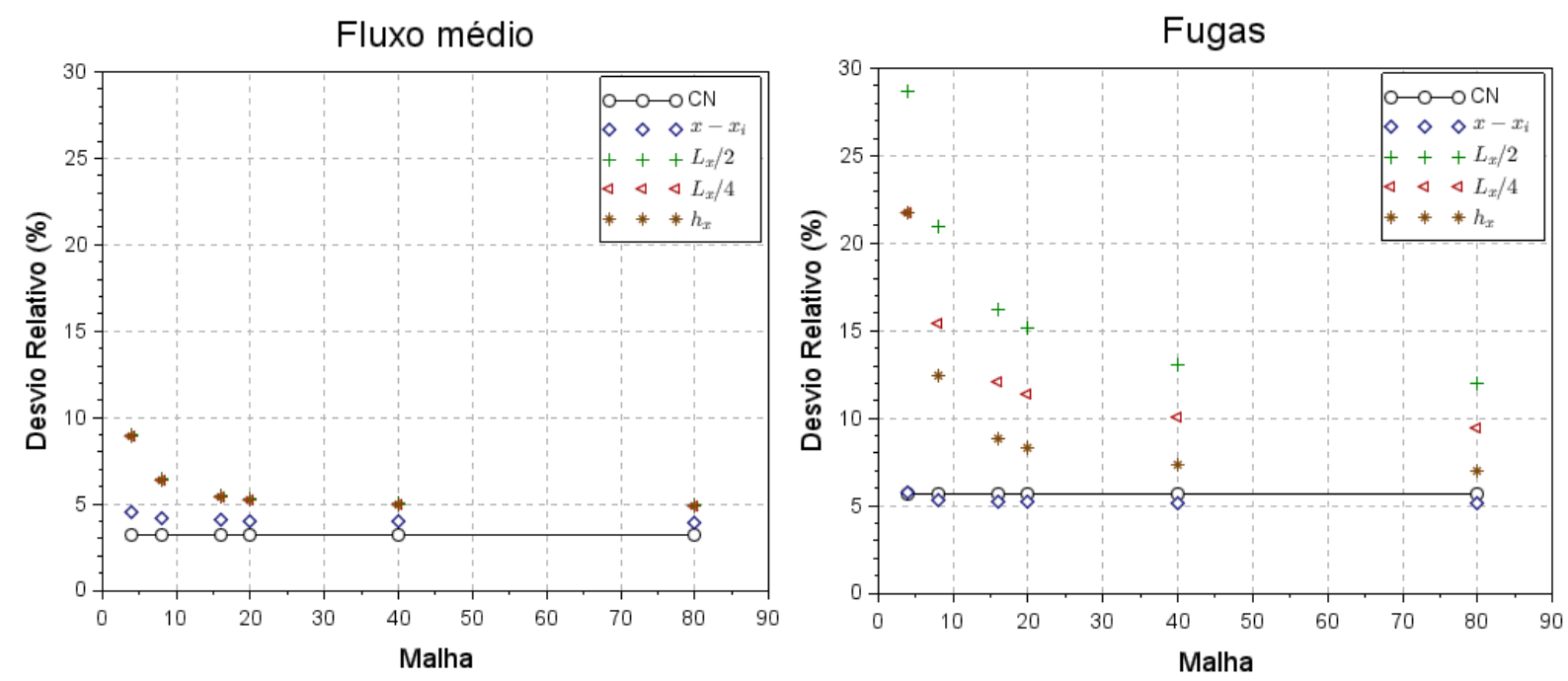

Figura 3. Desvios relativos para as quatro aproximações do termo exponencial com grade de uma região 
Da análise da Tabela 2 e da Figura 3, podemos constatar que um aumento no número de nodos da malha fina conduz a uma melhora na precisão dos resultados, o que ilustra que os erros de aproximação dependem dos parâmetros desta malha. Ao compararmos as aproximações propostas para o termo exponencial observamos que a aproximação $\left(x-x_{i}\right)$ é a que oferece os melhores resultados com erros na ordem de $5 \%$ para todas as malhas. Adicionalmente, observamos que esta aproximação do termo exponencial (1) oferece melhor desempenho computacional que as outras 3 (2, 3 e 4), precisando de menos iterações para alcançar a convergência. No caso das fugas de nêutrons o método CSG-SGF-Exp com a referida aproximação produzem resultados mais precisos que o método CSG-SGF-CN. Observamos um maior número de iterações no método exponencial se comparado ao método $\mathrm{CN}$, entretanto o método exponencial é mais preciso, vantagem que deverá ser mais marcada na resolução de problemas heterogêneos com elevados gradientes de fluxo neutrônico.

Considerando a aproximação $\left(x-x_{i}\right.$ ) para o termo exponencial foram realizados novos experimentos numéricos agora utilizando quatro nodos na grade grossa L, os resultados destes experimentos são oferecidos na Tabela 3. Na Figura 4 comparamos os desvios relativos para as duas malhas grossas consideradas $\mathrm{L}=1 \mathrm{e} \mathrm{L}=4$.

Tabela 3. Resultados numéricos do problema modelo para uma grade grossa de quatro regiões.

\begin{tabular}{|c|c|c|c|c|c|}
\hline Malha & Aprox. Exp. & $\bar{f}_{R}$ & $J_{D}$ & $J_{S}$ & Iterações \\
\hline \multirow{2}{*}{$4 \times 4 \times 4$} & $\left(x-x_{i}\right)$ & $1,3394 \mathrm{E}+01$ & $6,7909 E+01$ & $6,8034 \mathrm{E}+01$ & 27 \\
\hline & $\mathrm{CN}$ & $1,3068 \mathrm{E}+01$ & $6,9324 \mathrm{E}+01$ & $6,9324 \mathrm{E}+01$ & 26 \\
\hline \multirow{2}{*}{$4 \times 8 \times 8$} & $\left(x-x_{i}\right)$ & $1,3321 E+01$ & $6,8412 \mathrm{E}+01$ & $6,8562 \mathrm{E}+01$ & 33 \\
\hline & $\mathrm{CN}$ & $1,3068 \mathrm{E}+01$ & $6,9324 \mathrm{E}+01$ & $6,9324 \mathrm{E}+01$ & 33 \\
\hline \multirow{2}{*}{$4 \times 16 \times 16$} & $\left(x-x_{i}\right)$ & $1,3296 E+01$ & $6,9381 E+01$ & $6,9393 E+01$ & 41 \\
\hline & $\mathrm{CN}$ & $1,3068 \mathrm{E}+01$ & $6,9324 \mathrm{E}+01$ & $6,9324 \mathrm{E}+01$ & 41 \\
\hline \multirow{2}{*}{$4 \times 20 \times 20$} & $\left(x-x_{i}\right)$ & 1,3292E+01 & $6,9424 \mathrm{E}+01$ & $6,9431 E+01$ & 43 \\
\hline & $\mathrm{CN}$ & $1,3068 E+01$ & $6,9324 \mathrm{E}+01$ & $6,9324 \mathrm{E}+01$ & 43 \\
\hline \multirow{2}{*}{$4 \times 40 \times 40$} & $\left(x-x_{i}\right)$ & $1,3285 E+01$ & $6,9903 E+01$ & $6,9903 E+01$ & 49 \\
\hline & $\mathrm{CN}$ & $1,3068 E+01$ & $6,9324 \mathrm{E}+01$ & $6,9324 \mathrm{E}+01$ & 50 \\
\hline \multirow{2}{*}{$4 \times 80 \times 80$} & $\left(x-x_{i}\right)$ & $1,3281 E+01$ & $7,0538 E+01$ & $7,0537 E+01$ & 54 \\
\hline & $\mathrm{CN}$ & $1,3068 \mathrm{E}+01$ & $6,9324 \mathrm{E}+01$ & $6,9324 \mathrm{E}+01$ & 54 \\
\hline- & Referência & $1,3034 E+01$ & $7,1753 \mathrm{E}+01$ & $7,1753 \mathrm{E}+01$ & - \\
\hline
\end{tabular}


$4 \times 4 \times 4$ - 4 células na malha grossa , 4 células na malha fina em $x, 4$ células na malha fina em $y$

$\left(x-x_{i}\right)$, - aproximação do termo exponencial da Tabela 1

$\overline{f_{R}}$ - fluxo escalar de nêutrons na região $\mathrm{R}$

$J_{D}, J_{S}$ - corrente de nêutrons (fugas) nas fronteiras direita (D) e superior (S)

$\mathrm{CN}$ - resultados gerados pelo método CSG-SGF-CN

Referência - valor de referência obtido pelos métodos LN e SGF-LN
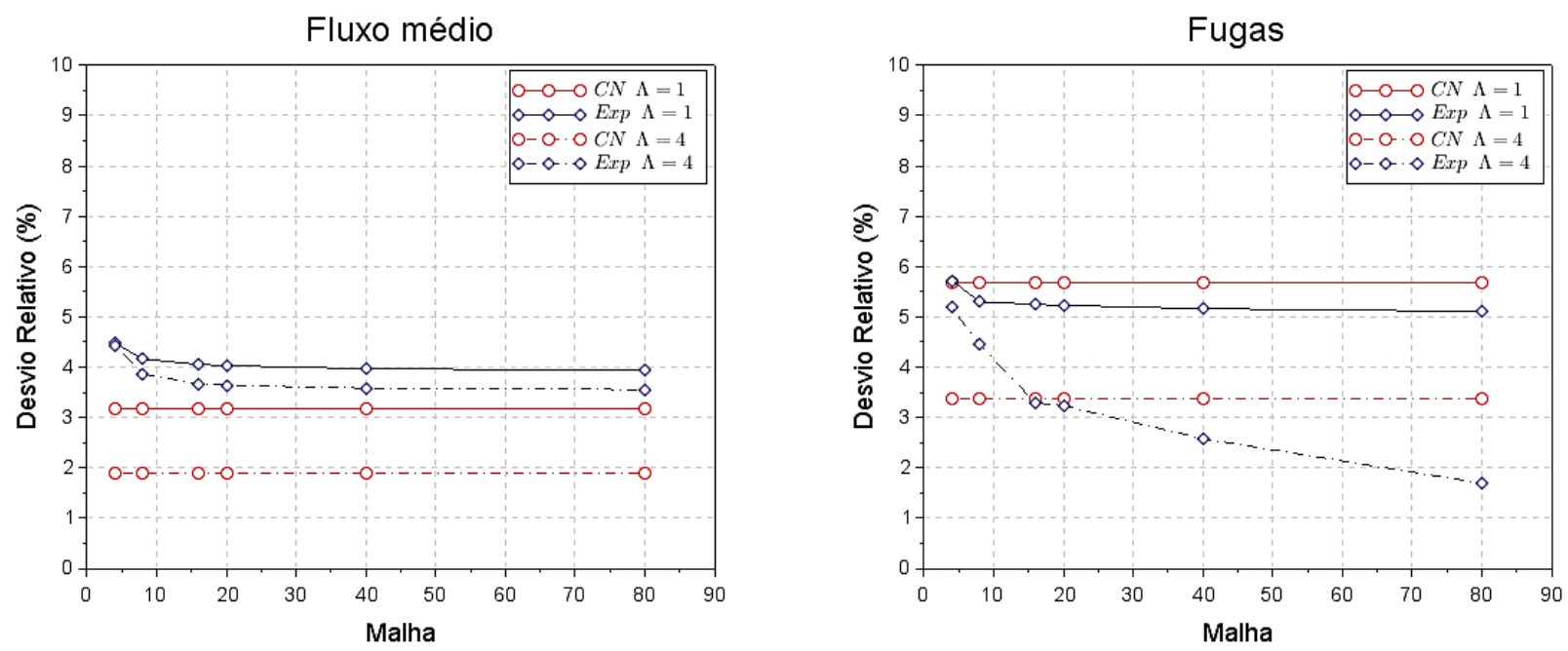

Figura 4. Desvios relativos dos métodos CSG-SGF-Exp (Azul) e CSG-SGF-CN (Vermelho) para malhas grosas de 1 e 4 regiões.

Ao compararmos as Tabelas 2 e 3, notamos que um refinamento na malha grossa nos leva a uma melhoria na precisão dos resultados. Esta característica é comum aos métodos de grades compostas, uma vez que a aproximação dos termos de fuga transversal é feita a nível de região. Conforme esperado, o refinamento da malha grossa produz uma queda acentuada no desempenho computacional. Novamente, observamos que para o método exponencial o refinamento da malha fina melhora a precisão dos resultados, como este comportamento não aparece no método com aproximação constante, podemos afirmar que a instabilidade numérica tem maior influência nos resultados gerados pelo método CSG-SGF-Exp.

\section{CONSIDERAÇÖES FINAIS}

Neste trabalho apresentamos os fundamentos matemáticos do método espectro nodal de grades compostas com aproximação exponencial para domínios homogêneos. 
Quatro alternativas de aproximação do termo exponencial são exploradas mediante a resolução de um problema modelo.

A partir dos resultados obtidos podemos afirmar que o método CSG-SGF-Exp oferece resultados satisfatórios em problemas homogêneos, a precisão dos resultados aumenta com a diminuição das dimensões da grade externa (grossa) e das grades internas (finas). Das aproximações verificadas para o termo exponencial, $\left(x-x_{i}\right)$ oferece os melhores resultados considerando a precisão e o desempenho computacional. 0 método proposto mostrou-se sensível ao fenômeno de instabilidade numérica o que não ocorre nos métodos de grades compostas desenvolvidos anteriormente.

Os desdobramentos futuros desta pesquisa envolvem a extensão e validação do método para domínios heterogêneos, onde o fluxo de nêutrons sofre fortes variações nas interfaces das regiões, nestes casos os resultados do método CSG-SGF-Exp devem ser muito mais precisos que os gerados pelo método CSG-SGF-CN.

\section{AGRADECIMENTOS}

Os autores do trabalho agradecem a Fundação de Amparo à Pesquisa do Estado da Bahia (FAPESB) pelo suporte financeiro parcial oferecido para execução do presente trabalho.

\section{REFERÉNCIAS}

ARNOLD, Vladimir. Ordinary Differential Equations. New York: Springer, 1992.

BADRUZZAMAN, A. Nodal methods in transport theory. Advances in Nuclear Science and Technology, vol. 21. New York: Plenum Press, 1990. p. 293-331.

BARROS, Ricardo C.; LARSEN, Edward. A spectral nodal method for one-group x,ygeometry discrete ordinates problems. Nuclear Science and Engineering, v. 111, p. 3445, 1992. .

BIRKHOFF, Garrett; VARGA, Richard S.; YOUNG, David. Alternating Direction Implicit Methods. In: ALT, Franz L.; RUBINOFF, Morris (org.). Advances in Computers,. [s.I.]: Elsevier, 1962. p. 189-273. DOI 10.1016/S0065-2458(08)60620-8. Disponível em: https://linkinghub.elsevier.com/retrieve/pii/S0065245808606208

CARLSON, B. G.; LATHROP, K. D. Transport theory - the method of discrete ordinates. In: GREENSPAN, H.; KELBER, C. N.; D., Okrent (org.). Computing Methods in Reactor Physics. New York: Gordon \& Breach, 1968. 
CASE, Kenneth M.; ZWEIFEL, Paul F. Linear Transport Theory. Reading, Massachussetts: Addison - Wesley, 1967.

DE MELLO, José Antonio M.; BARROS, Ricardo C. An exponential spectral nodal method for one-speed $\mathrm{X}, \mathrm{Y}$-geometry deep penetration discrete ordinates problems. Annals of Nuclear Energy, v. 29, n. 15, p. 1855-1869, out. 2002. DOI 10.1016/S03064549(02)00013-0. .

DOMINGUEZ, D.S.; HERNANDEZ, C.R.G.; BARROS, R.C. Spectral nodal method for numerically solving two-energy group $X, Y$ geometry neutron diffusion eigenvalue problems. International Journal of Nuclear Energy Science and Technology, v. 5, n. 1, 2010. DOI 10.1504/IJNEST.2010.030307. .

DOMINGUEZ, Dany S. et al. A composite spatial grid spectral Green's function method for one speed discrete ordinates eigenvalue problems in two-dimensional Cartesian geometry. Progress in Nuclear Energy, v. 109, p. 180-187, nov. 2018. DOI 10.1016/j.pnucene.2018.08.007. Disponível em: https://linkinghub.elsevier.com/retrieve/pii/S0149197018301896.

DOMINGUEZ, Dany S.; BARROS, Ricardo C. The spectral Green's function linear-nodal method for one-speed $\mathrm{X}, \mathrm{Y}$-geometry discrete ordinates deep penetration problems.

Annals of Nuclear Energy, v. 34, n. 12, p. 958-966, dez. 2007. DOI 10.1016/j.anucene.2007.04.015. .

DOMINGUEZ, Dany S et al. Composite spatial grid spectral nodal method for one-speed discrete ordinates deep penetration problems in X, Y geometry. Progress in Nuclear Energy, v. 52, p. 298-303, 2010. DOl 10.1016/j.pnucene.2009.04.004. .

FRANKLE, C.M.; DALE, G.E. Unconventional neutron sources for oil well logging. Nuclear Instruments and Methods in Physics Research Section A: Accelerators, Spectrometers, Detectors and Associated Equipment, v. 723, p. 24-29, set. 2013. DOI 10.1016/j.nima.2013.05.012. Disponível em:

http://www.sciencedirect.com/science/article/pii/S0168900213005378. Acesso em: 8 jul. 2015.

LEWIS, E. E.; W. F. MILLER, Jr. Computational Methods of Neutron Transport. 1. ed. New York: Jhon Wiley \& Sons, 1984.

MOSS, Raymond L. Critical review, with an optimistic outlook, on Boron Neutron Capture Therapy (BNCT). Applied radiation and isotopes : including data, instrumentation and methods for use in agriculture, industry and medicine, v. 88, p. 2-11, jun. 2014. DOI 10.1016/j.apradiso.2013.11.109. Disponível em:

http://www.sciencedirect.com/science/article/pii/S0969804313005587. Acesso em: 30 mar. 2015.

NASCIMENTO, Hugo M.; DOMINGUEZ, Dany S. Método Espectro Nodal de Grades Compostas para Problemas de Autovalor de Difusão. Saarbrucken, Deutscland: Verlag / Novas Edições Académicas, 2015. 
DOI: $10.18605 / 2175-7275 /$ cereus.v12n3p99-115

Revista Cereus

2020 Vol. 12. N.3
AGUIAR, A.S. O; DOMINGUEZ, D.S; IGLESIAS, S.M; HERNANDEZ, C.R.G.

Método Espectro Nodal de Grades Compostas com Aproximação Exponencial na Formulação de Ordenadas Discretas em Meios Homogêneos Não-multiplicativos.

Problemas de Fonte Fixa na Formulação de Ordenadas Discretas a um Grupo de

Energia em Geometria Cartesiana Bidimensional. 2016. 78 f. Universidade Estadual de Santa Cruz, 2016. 